\title{
Characterization of vulnerability of road networks to fluvial flooding using SIS network diffusion model
}

\author{
Bahrulla Abdulla ${ }^{1 *}$, Amin Kiaghadi $^{2}$, Hanadi S. Rifai $^{3}$ and Bjorn Birgisson ${ }^{1}$
}

\begin{abstract}
This study aims to characterize the vulnerability of road networks to fluvial flooding using a network diffusion-based method. Various network diffusion models have been applied widely for modeling the spreading of contagious diseases or capturing opinion dynamics in social networks. By comparison, their application in the context of physical infrastructure networks has just started to gain some momentum, although physical infrastructure networks also exhibit diffusion-like phenomena under certain stressors. This study applies a susceptible-impacted-susceptible (SIS) diffusion model to capture the impact of flooding on the road network connectivity. To that end, this paper undertook the following four steps. First, the road network was modeled as primal graphs and nodes that were flood-prone (or the origins of the fluvial flood) were identified. Second, temporal changes in the flood depth within the road network during a flooding event were obtained using a data-driven geospatial model. Third, based on the relationship between vehicle speed and flood depth on road networks, at each time step, the nodes in the road network were divided into two discrete categories, namely functional and closed, standing for Susceptible and Impacted in the SIS diffusion model, respectively. Then, two parameters of the SIS model, average transition probabilities between states, were estimated using the results of the hydraulic simulation. Fourth, the robustness of the road network under various SIS diffusion scenarios was estimated, which was used to test the statistical significance of the difference between the robustness of the road network against diffusions started from the randomly chosen nodes and nodes with different high centrality measures. The methodology was demonstrated using the road network in the Memorial super neighborhood in Houston. The results show that diffusive disruptions that start from nodes with high centrality values do not necessarily cause a more significant loss to the connectivity of the road network. The proposed method has important implications for applying link predictions on road networks, and it casts significant insights into the mechanism by which cascading disruptions spread from flood control infrastructure to road networks, as well as the diffusion process in the road networks.
\end{abstract}

Keywords: Network diffusion, Giant connected component (GCC), Network centrality measures, Cascading failures

\section{Introduction}

Changes in the earth climate, potential global warming, and unprecedented and ever-increasing urbanization, coupled with the increased interdependence among different sectors, are putting critical infrastructure systems

\footnotetext{
* Correspondence: babdulla@tamu.edu

'Zachry Department of Civil Engineering, Texas A\&M University, 3136 TAMU, College Station, TX 77843, USA

Full list of author information is available at the end of the article
}

under increasing pressure [26]. In the meantime, failures in critical infrastructure systems are becoming prohibitively costly, mainly due to the possible cascading failures that are initiated from one sector and subsequently cause a series of failures in other dependent sectors. Thus, the resilience of interdependent critical infrastructure (ICI) systems is one of the grand challenges facing engineers and policy-makers in the twenty-first century $[13,22,33]$. Over the past two decades, the body of

\section{Springer Open}

(c) The Author(s). 2020 Open Access This article is licensed under a Creative Commons Attribution 4.0 International License, which permits use, sharing, adaptation, distribution and reproduction in any medium or format, as long as you give appropriate credit to the original author(s) and the source, provide a link to the Creative Commons licence, and indicate if changes were made. The images or other third party material in this article are included in the article's Creative Commons licence, unless indicated otherwise in a credit line to the material. If material is not included in the article's Creative Commons licence and your intended use is not permitted by statutory regulation or exceeds the permitted use, you will need to obtain permission directly from the copyright holder. To view a copy of this licence, visit http://creativecommons.org/licenses/by/4.0/. 
knowledge on ICI resilience has advanced in the domains of modeling, simulation methods, and theoretical frameworks. Despite the growing literature [10, 12, 25] on ICI resilience, our understanding of the dynamics and mechanisms of disruptions in ICI systems that shape resilience patterns in these complex networks is somewhat limited. This lack of understanding is particularly evident in urban areas where transportation systems are frequently affected by weather-related hazards.

Flooding, especially ones due to excessive and intense rainfall precipitation, has been the predominant cause of weather-related disruptions to the transportation infrastructure [23]. Such events could undermine the vital functionality of transportation systems, especially road networks. Many studies have shown that roads are among the major causes of deaths in cities during flooding; this is mainly due to the vehicles being driven through flooded roadways $[3,9,11,16]$. Locations, such as Texas, where road mobility through cars is the primary mode of passenger transportation, are especially vulnerable to the impact of flooding [5]. The advantage of having one of the largest road networks in the U.S. could become a curse when the majority of the roads are closed due to flooding and there are few other alternatives to go around the city, as was the case during Hurricane Harvey in 2017 [2]. In addition, during disastrous events, the road network functions as a lifeline system for rescuing people and assets and plays a vital role in repairing and restoring other infrastructure systems when they are disrupted. In order to cope with disruptions efficiently and take active precautionary measures, it is critical to understand the mechanisms and patterns with which the disruptions unfold in the transportation network. Due to the planar nature of transportation networks, they tend to lend themselves readily to being represented as graphs, and therefore graph theory-based approaches have been one of the standard tools to study the vulnerability in transportation systems [32]. Graph theory reduces a road network to a mathematical matrix where the vertices (nodes) represent road intersections and the edges are the road sections between these nodes [18]. This type of matrix abstraction of road networks not only facilitates the accessibility and connectivity analysis but also assists in the identification of critical locations using available graph-theoretic centrality measures. However, there are two crucial challenges in network modeling of transportation networks. On the one hand, transportation networks, similar to many other critical infrastructure networks, are spatially embedded [4] and the configurations of the environment in which network elements (nodes or edges) operate are inherently heterogeneous. This fact, when coupled with the possible spatial or temporal variance of the magnitude of the disruptive events, makes failure probabilities significantly variable from node to node. On the other hand, the topology of most critical infrastructure networks is intrinsically dynamic and evolving, especially during disruptive events. Understanding the patterns for temporal shifts in the functional topology of critical infrastructure networks during disastrous events is a crucial step in devising efficient plans to reduce their vulnerabilities. However. the almost complete absence of the time dimension in such problem definitions is a problem that can be attributed to (1) the graph theory ancestry of the field, and (2) the limited number of dynamic data sources available when the area of complex networks analysis emerged [27].

Flooding in urban roadways is a process that presents both of the challenges mentioned above. The floodinduced disruption to the road network is realized by rendering certain components of the roadway system non-functional. For example, certain road sections or intersections could suffer from high water levels and be forced to be closed. Another important disruption mechanism of floods to the road network is the scouring of bridges [7, 19, 34], which can cause both short-term or long-term damage to road network connectivity. Relevant studies in the literature that are aimed at tackling the flood vulnerability of critical infrastructure networks could be categorized into two main types: (1) graphtheory based topological approaches that focus on topological integrity of the network; and (2) hydrological approaches that model the flood propagation process in (or around) critical infrastructure in urban areas [31]. Each of these methods considers the flood vulnerability problem from different angles; consequently, each approach only reflects some parts of the whole picture. Most of the studies attempted to apply dynamic network modeling approaches focused on complete or random graphs to demonstrate their applicability in real-world network failure problems. However, transportation networks are neither random nor complete. They have a unique configuration manifested in a relatively small range of node-degrees and spatial constraints that are not observed in other types of networks. The aforementioned historic decoupling between the two types of methods could largely be attributed to the lack of granular flood data that could be input to network models.

Recently, for identifying the probability of flooding in a road network, the coupling of remotely sensed data with hydrodynamic models has been used. Such an approach was used to identify the most critical and vulnerable nodes (intersections) in a transportation network. Sadler et al. [28] combined storm surge levels associated with different return periods, provided by the Federal Emergency Management Administration (FEMA), with High-resolution Digital Elevation Models (DEMs), compiled from data collected by Light Detection and 
Ranging (LiDAR). The authors then compared the surge elevations with the road elevations to assess different scenarios and reported the most vulnerable roadway segments based on the frequency of flooding. In another study, Kalantari et al. [14] developed a LiDAR-based data-driven model to quantify the risk of flooding and sediment transport at different roadway intersections in Sweden. While these efforts are essential to study the impacts of the most severe inundation scenarios, they do not provide enough information on how the internal components of the system behave during a flood event. This limitation is mainly due to the use of only one snapshot of the flood rather than a time series of water depth. In contrast, other researchers have coupled the results of hydrodynamic models with remotely sensed elevation data to estimate the probability of heavy inundation during flooding events. Courty et al. [8], Lagmay et al. [17], and Pyatkova et al. [24] coupled the results of MIKE FLOOD, LISFLOOD-FP, and FLO-2D GDS PRO, respectively, to LiDAR elevations and reported the risk of inundation for roads during flooding events. Though more accurate hydrodynamic models are useful tools in storm surge and flood simulation/prediction, they are costly because of lengthy computational time, expensive equipment, and the need for skilled users. In addition, an extensive calibration of the model using observed data is required to enhance model reliability. In summary, existing methods are either focused on a single point in the duration of the disastrous event and there is a lack of understanding about the internal mechanism of the disruptive events on road networks; or are computationally or operationally too expensive. This study aims to bridge the gaps mentioned above between these two closely related fields. Furthermore, given the improved computational powers and relatively wide availability of the data, the condition is mature enough to do a more granular and detailed temporal analysis on the road network. This study is motivated by these factors; in the study, a simple methodology was developed to have both the reliability of using field measured data directly and the advantage of using time series water depth instead of one snapshot. To be more specific, the measured highwater marks (HWMs) after a flooding event were combined with the observed pattern in water surface elevation (WSE) of nearby rivers recorded by the United States Geological Survey (USGS) to create a WSE time series at the location of the HWMs. Each time series was then compared with LiDAR elevations to calculate the water depth at any given point.

When it comes to analyzing the effect of flooding on network vulnerability, it is important to know how a phenomenon spreads through a network. Based on authors' interviews with stakeholders of critical infrastructure systems in Houston, after runoff conveyance infrastructure systems (bayous, channels, creeks, and stormwater systems) reach their capacity under an excessive rainfall, road networks become part of the conveyance system and play the role of moving excessive water into lower elevation areas and/or releasing water into other storm-water drainage systems. In this context, the process of spreading the floodwater around the road network could be assumed as a diffusion process, which is analogous to the spread of contagious diseases among human beings. The origin of diffusion modeling could be traced back to the spread of epidemics and mathematical modeling of epidemics predates most of the studies on networks by many years [20]. The traditional diffusion modeling approaches avoid discussing contact networks by making use of fully mixed or mass-action approximation, in which it is assumed that every individual (node in the network) has an equal chance, per unit of time, of coming into contact with every other node [21]. Based on the assumptions of this approach, nodes (people) mingle and meet completely at random, which is not a realistic representation of most real-world networks. This un-realistic representation is because nodes in real-world networks are spatially embedded and have a heterogeneous exposure to diffusion mechanisms (the reader is referred to Shakarian et al. [30] for a comprehensive review of network diffusion). In summary, the majority of the research in the field of network vulnerability is focused on theoretical networks; a fewer number of published research papers are focused on realworld networks. Due to their unique topological structure and configuration, road networks represent one unique type of real-world networks. Understanding, characterizing, and conceptualizing these networks could bridge the gap between advancement in the field of theoretical networks and real-world networks. The proposed method facilitates the assessment of the vulnerability of the road network to flooding which contributes to the advancement of network science in the realm of real-life networks. Given that flooding and inundation of road networks occurs frequently and all around the globe, the findings from this research are directly applicable to other road networks and of interest to many.

\section{Methodology}

A summary of the methodology used in the study is presented in Fig. 1. The first step was modeling the road systems as the primal graph, which is followed by a simulation of the hydraulic process in the areas where roads are located in order to obtain the granular (nodelevel) flood depth data. A diffusion model that is commonly used to study the spread of communicable disease, Susceptible-Impacted-Susceptible (SIS Model), is proposed to model the propagation of the flood in road 


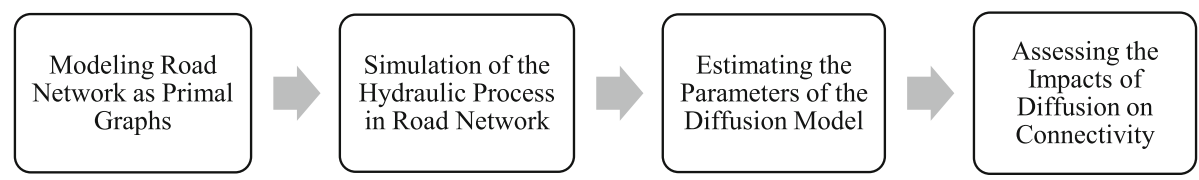

Fig. 1 Research Methodology

networks. Parameters of the SIS model were estimated using the temporal flood depth for the nodes in the road network. Finally, the impact of both the number and locations of the seed nodes on the connectivity of the road networks during diffusion was evaluated.

\section{Road network Modelling}

The road network was modeled as a non-planar primal graph, where nodes represent the intersections in the road network while edges represent the actual road sections. As a proxy for flood vulnerability, the elevation of each node in the road network was also retrieved using Google Application Programming Interfaces (API). Road network topological data and other auxiliary information were obtained from OpenStreetMap using the OSMnx python package [6]. As the vulnerability of roads that used for vehicular travel is the primary focus of this study, we chose to focus on road types that are used for passenger and service vehicles and didn't include the roadways intended for biking or walking, which is also available through OSMnx.

\section{Simulation of the hydraulic process}

In this study, the depth of flooding at nodes within the road network was used as a proxy for their functional status. Therefore, obtaining the granular temporal flood depth information in the road network during the case study event- Hurricane Harvey, was crucial. During Harvey, flooding in the study started at 20:00:00 on August 26, 2017. The temporal changes in the flood depth at the node location in the road network was obtained for a temporal scale of 17 days, observations are in hourly intervals, from 12 AM, 25 August 2017 to 11 PM, 10 September 2017. This study looked into the time between 22:00:00 on August 26, 2017, and above peak period, which is 11:00:00 on August 30, 2017.

The methodology applied in this study to calculate the water depth at each node of the road network is similar to what Kiaghadi et al. [15] developed. In brief, a geospatial model was developed in ArcMap using the many existing tools including Feature to Polygon, Intersect, Topo to Raster, Extract by Mask, Mosaic to New Raster, Resample, and Raster Calculator. Catchment shapefiles and HWMs points were used in the developed mode as inputs to generate a continuous WSE raster at $1 \mathrm{~m}$ by 1 $m$ resolution were generated. The land elevation represented by the LiDAR DEM raster was then subtracted from the continuous WSE raster to develop the inundation raster with depth information at the desired resolution. To isolate the effect of flooding existing waterbodies were eliminated from the generated inundation raster. The final product was used as a static snapshot of the event that represents the worst-case flooding scenario. The main difference was converting the observed HWMs (one snapshot of the flood representing the maximum WSE) into a time series. In other words, in the current study, a water surface elevation over the time of the flooding event (i.e. Hurricane Harvey) was used instead of a static snapshot of the event. Due to a smaller study area, all calculations were undertaken at the catchment level and only HWMs within the catchments covering the study area were used. Catchment boundaries were extracted from the watershed delineation in the Tropical Storm Allison Recovery Project (TSARP). Figure 2 shows the catchments and associated HWMs used in the study. A total of 11 HWMs were used.

To convert the single measured HWM values into a WSE time series, the observed temporal pattern in the WSE at the closest USGS gage was used. Since the majority of the HWMs were measured close to the banks of rivers and were caused by the river overtopping its banks, it was assumed that the WSE time series at the location of the HWM was similar to the river behavior. The HWM represents the highest level of water observed at the specific location that is equivalent to the peak of the WSE time-series recorded by USGS. For the period of simulation and for each USGS gage, the ratio between the WSE at each time step and the peak were calculated and multiplied by the reported values of nearby HWMs to generate the WSE time series at the location of each HWM. For HWMs located on the tributaries (see HWM2 in Fig. 2), the pattern observed in the difference between recorded discharges from two USGS gages (one upstream and one downstream) was applied to the HWMs. Here, it was assumed that the difference in the discharge rates was solely caused by the input from the tributary and not by direct runoff from the drainage areas between the two USGS gages. To automate the process of generating a WSE at each time step (1 h), a model was built in ArcMap. Several existing tools in ArcMap were applied to (1) Convert the HWMs within the catchments into a WSE raster with a resolution of $1 \mathrm{~m}$ by $1 \mathrm{~m}$ for each time step; (2) Subtract the 


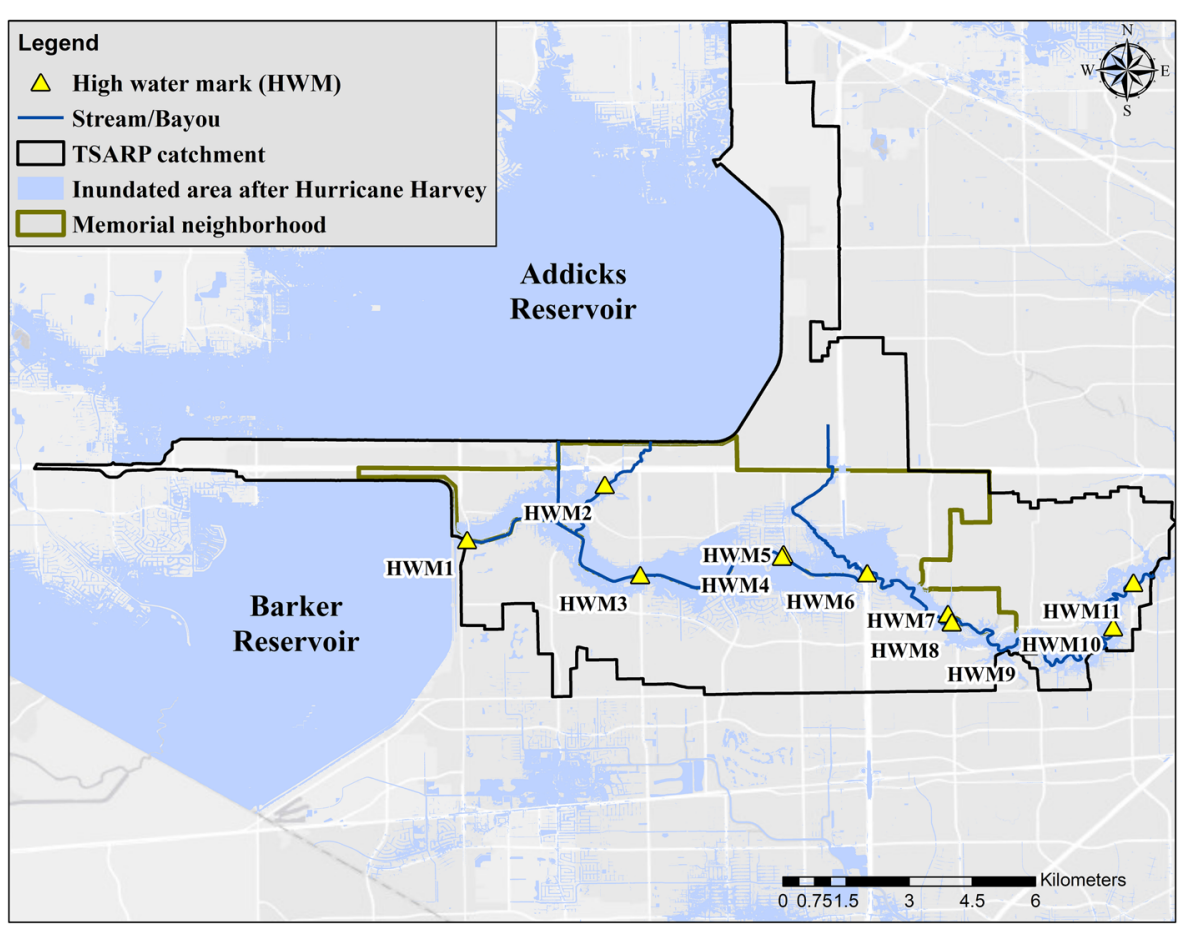

Fig. 2 Study area and location of High-Water Marks (HWMs) used in the study

surface elevation raster (LiDAR DEM) from the WSE raster to calculate the water depth at time steps; (3) Extract the water depths at the locations of specific nodes (intersections) for each time step; (4) Filter the depths to only consider nodes with a positive depth. A negative value indicates that the river water is contained within the original river bank; (5) Export the excel file containing the locations and associated water depths. A MATLAB code was developed to combine all the excel files and create a metafile with the locations of the nodes and water depth at each time step over the length of the simulation.

\section{Estimation of the parameters of the SIS network diffusion model}

It was hypothesized that the propagation of the flooding impacts in the road network can be modeled using the SIS diffusion approach. Based on a separate study in which authors proposed a Bayes-rule based percolation approach for the road network during flooding [1], highly flood-prone areas tend to be inundated first due to overflow of flood control infrastructure. The disruption propagates to adjacent areas based on factors like relative elevation, drainage condition, terrain, the level and type of vegetation, soil type, intensity and duration of rainfall, among many others. Due to the complex and stochastic nature of the propagation of the floods, a probability-based approach should be used to model its disruptions on the road network. At the propagation stage, due mainly to gravity, a node is more likely to be inundated because of the existence of an inundated adjacent node. The same above factors might also cause the receding of the flood at a certain location, which makes that particular node functional again. The binary transitions between the functional or non-functional statues of the nodes in the road network could be captured using a basic diffusion model called SIS diffusion. In SIS diffusion, there are two types of nodes (Susceptible and Infected), and the rate of change between these two statuses is characterized by two parameters labeled beta and gamma (See Fig. 3). The main reason to focus on the nodes, instead of links is because once a node is flooded enough to be removed from the network, the edges connected to that node will be rendered as nonfunctional and will no longer be part of the connected network component.

The first task in developing the SIS diffusion model is to categorize the study population (nodes in this context) into different classes using certain criteria defined by the user. In the context of the current study, it is appropriate to categorize the nodes in the road network into functional or closed categories. During a flooding event, the closed or functional status of the road network is a binary value, but the flooding (depth) status in different parts of the road network is a continuous variable. It is possible to relate the flood depth to the closed or functional status of roads via vehicle speed. Researchers have studied the relationship between the 


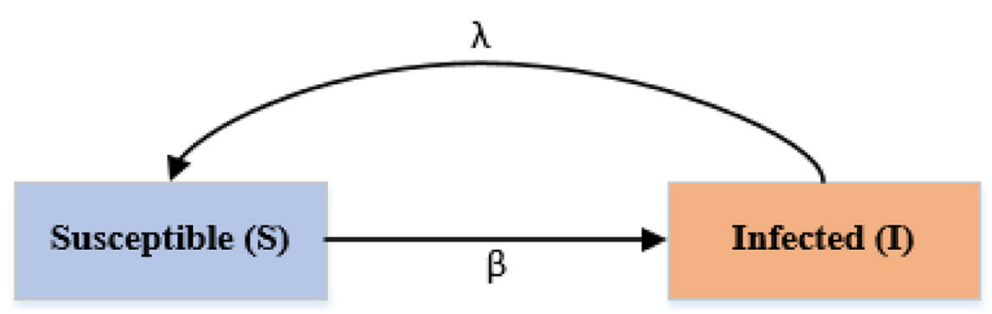

Fig. 3 Schematic representation of the SIS Diffusion Model

depth of the flooding and the speed of the vehicles driving on the roads during the flooding event. Pregnolato et al. [23] have estimated the relationship between the depth of standing water and the speed of different types of vehicles as:

$$
v(w)=0.0009 w^{2}-0.5529 w+86.9448
$$

where:

$\mathrm{v}(\mathrm{w})$ is the vehicle speed $(\mathrm{km} / \mathrm{h})$, and $\mathrm{w}$ is the depth of the floodwater on the road(in $\mathrm{mm}$ ).

Using Eq. (1), nodes in the road network at any given point in time were divided into two categories based on the vehicle speed. (1) Susceptible Nodes (S): The susceptible group is a node in the network that is either intact at a given point or the flood depth in the node location is less than $140 \mathrm{~mm}$. On such types of nodes, the passenger vehicle speed is more than or equal to $20 \mathrm{~km} / \mathrm{h}$. (2) Infected Nodes (I): The Impacted group is the nodes that have been heavily impacted by the flood and are rendered non-functional. The speed of vehicles on these types of nodes is less than $20 \mathrm{~km} / \mathrm{h}$. The next step in SIS diffusion modeling is to identify the seed nodes, the portion of the nodes in the network that were already impacted when diffusion started. During a fluvial (or riverine) flooding event, the nodes located within the flood-prone areas initiate a flood-induced diffusion phenomenon in the road network. Estimating the floodproneness of the nodes can be based on the floodplain type, proximity to flood control infrastructure and relative elevation of the nodes [1]. The last step is to estimate the other two essential parameters of the SIS diffusion, $\beta$, and $\gamma$. Parameters of the diffusion model are solved for using eqs. (2) and (3) from Newman [21]:

$$
\begin{aligned}
& \frac{d S}{d t}=\gamma x-\beta s x \\
& x(t)=x_{0} \frac{(\beta-\gamma) e^{(\beta-\gamma) t}}{\beta-\gamma+\beta x_{0} e(\beta-\gamma) t}
\end{aligned}
$$

where:

$\beta$ and $\gamma$ : transition parameters of diffusion; S: number of susceptible individuals (nodes) at a given point in time; I: number of infected individuals (nodes) at a given point in time; $x(t)$ : the fraction of infected nodes at a given point in time; $x_{0}$ : the fraction of susceptible nodes at the beginning of diffusion.

\section{Assessing the diffusion profile and connectivity under different scenarios}

Connectivity profiles of the road network under two types of disruptions were studied. (1) The impacts of the flood-induced network diffusion that started at different locations on the connectivity profile of the same road network; (2) The impact of diffusion on the overall connectivity of the road network during the flood propagation process in the road network by studying the road network in all 88 neighborhoods in the case study area.

\section{Connectivity profile}

This study first estimated the parameters of SIS diffusion under different thresholds: $140 \mathrm{~mm}$ represents the maximum flood depth in which sedan cars can travel on the road while $300 \mathrm{~mm}$ represents the depth for SUV vehicles, whereas $600 \mathrm{~mm}$ represents the threshold flood depth for the fire trucks. Then using the SIS diffusion parameters under the $140 \mathrm{~mm}$ threshold, connectivity profile of the road network under different scenarios were examined. The diffusion scenario which starts from randomly selected seed nodes was considered as a baseline scenario. Due to diverse colocation patterns between road networks and flood control infrastructure networks, it is possible for the fluvial flooding to occur at any location in the road network. Because of the unique topography and layout of the road networks, nodes with high centrality measures represent unique locations on the road networks. The impacts of diffusion on road networks was quantified using the relative (to original network size) size of the giant connected component (GCC) [29] in the road network. Five considered scenarios include: (1) diffusion is initiated from a certain number of randomly selected nodes; (2) diffusion started from a certain number of nodes with the highest betweenness centrality (BC); (3) diffusion started from a certain number of nodes with the highest degree centrality (DC); (4) diffusion started from a certain number of nodes with 
highest closeness centrality (CC); (5) diffusion started from a certain number of nodes with highest eigenvector centrality. For this analysis, the road network in the Memorial super neighborhood was used in the analysis (Fig. 4). A super neighborhood in the case study region is geographically designated area in which different stakeholders work collectively to address the needs and concerns of the community.

\section{Overall connectivity}

While the connectivity profile could cast some insights into the sensitivity of diffusion at different levels on the connectivity of the road network, it is not an aggregate measure of the overall impact of flooding on the road connectivity. Therefore, a measure called overall connectivity $(\mathrm{OC})$ is introduced to assess the connectivity of the network during the diffusive disruptive events. The connectivity changes due to diffusive disruptions are quite uneven under different scenarios. In order to make the magnitude of the impacts of different diffusions on road network comparable, the area under the performance curve was calculated. OC is defined using Eq. 4 below:

$$
O C=\int_{t_{0}}^{t_{1}} G C(t) d t
$$

where:

$t_{0}$-the starting time for the disruptive event; $t_{1}$-the time the disruptive event ends; $G C(t)$-the relative size of the connected giant component in the road network.

In order to examine the impacts of the location of the initial diffusive set seeds on the vulnerability of the road network, a two-sample significance test was conducted. The working hypothesis was that the road network is

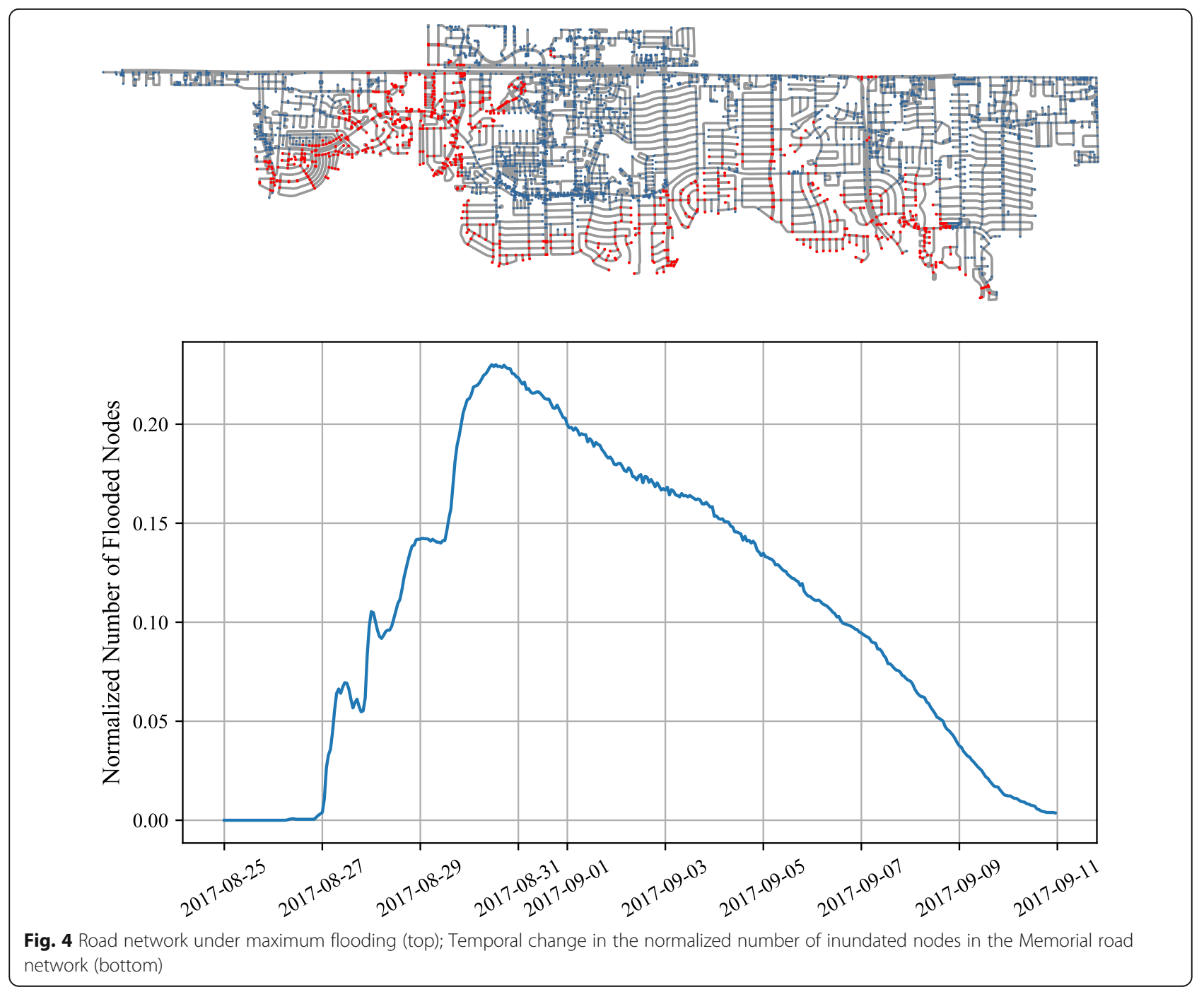


more vulnerable to the contagious disruptions that start from the significant nodes (with high centrality measures). This vulnerability is because the removal of the significant nodes alone usually caused a greater magnitude of loss to the connectivity of the network. If we classify road networks under the disruptions of random diffusive failures as group 1, road networks under the targeted diffusive failures (failures originating from those nodes which are considered significant, i.e. high degree centrality, high betweenness, nodes with low closeness centrality and nodes with high Eigenvector centrality) would be classified as group 2 . The overall connectivity of the network in the two groups was studied in this research as were the OC values for each of the 88 super neighborhoods in the study area.

\section{Results and discussion}

\section{Road network and hydraulic process}

The road network in the Memorial super neighborhood has 4073 nodes and 9762 edges, with an average node degree of 2.397. A snapshot of the road network when the most severe flooding occurred can be seen in Fig. 4, which happened at 11:00:00 on August 30th, 2017, when the maximum number of nodes (937 nodes out of 4073) flooded in the network. A flood depth observation for each node in the road network, at the hourly interval, was made for $408 \mathrm{~h}$. A temporal change in the fraction of flooded nodes (as long as a node is under non-zero flood water, it was considered as flooded) can be seen in Fig. 4. In Using the size of the giant connected component in the network as measure of overall connectivity, the temporal change in the performance of the network during the flooding, under different closure thresholds for node-removal (a condition that corresponds to road closure for different types of vehicles, $140 \mathrm{~mm}$-sedan cars, $300 \mathrm{~mm}$-SUVs, and $600 \mathrm{~mm}$-common fire trucks) was presented in Fig. 5. As could be seen in Fig. 5, the vulnerability of the road network connectivity under different thresholds is quite different. Understanding the mechanism behind the sudden drop in the relative size of the giant connected component at the $140 \mathrm{~mm}$ threshold has important implications for improving the resilience of the road network to flooding events.

\section{Obtaining the network diffusion parameters}

Three parameters were estimated for the SIS diffusion model. The initially impacted parameter was estimated based on the number of nodes within a certain type of flood plain. The transition rate parameters $(\beta$ and $\gamma)$ were estimated by minimizing the residual sum of the squares method. In other words, the sum of squares of the difference between predicted and observed node numbers in each category is minimized. Table 1 presents a summary of the parameter estimation for the SIS model under the four different flood threshold scenarios, that corresponds to maximum threshold for the flood depth for certain types of vehicle, as discussed in the methodology section of the paper.

In order to characterize the vulnerability of road network to various diffusive disruptions, two types of simulation experiments were conducted, as discussed in the methodology section.

\section{Experiment one: assessing the impact of diffusion on connectivity profile}

This study first estimated the parameters of the SIS diffusion based on the actual hydraulic process in the road network, which facilitated simulations of the road network

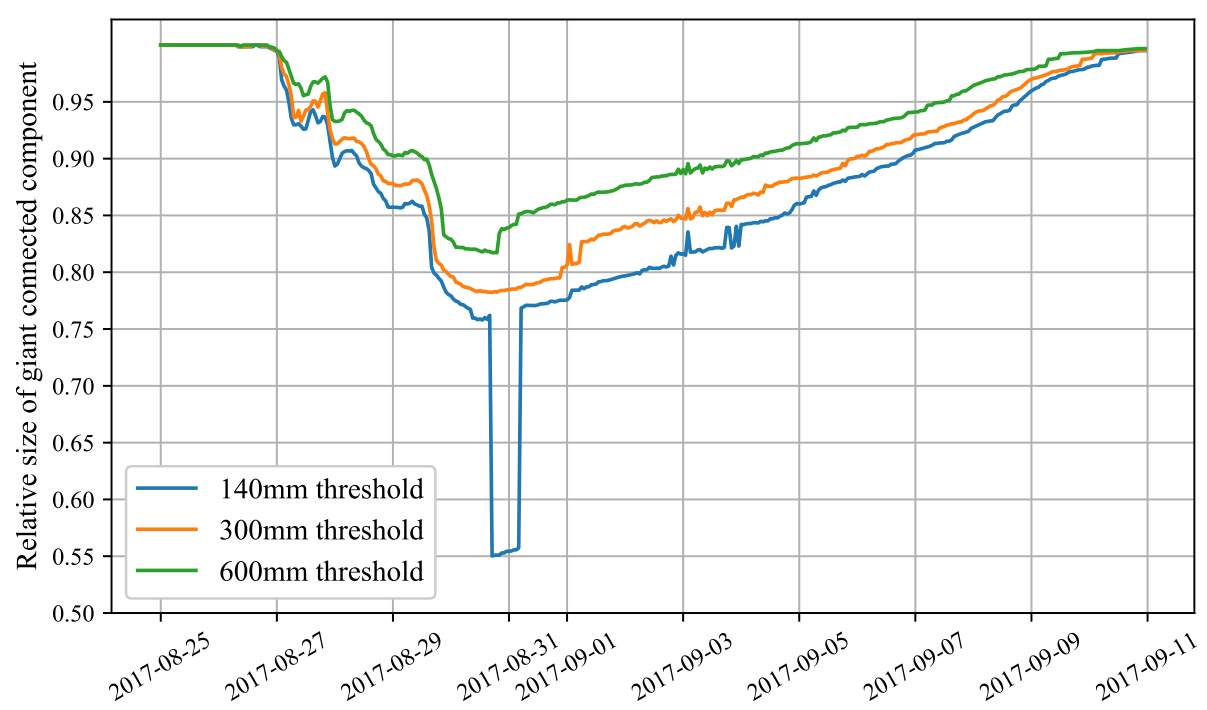

Fig. 5 Size of GCC in road network under different closure thresholds 
Table 1 Summary of diffusion parameters under different diffusion threshold

\begin{tabular}{llll}
\hline Diffusion Threshold (in mm) & Beta $(\boldsymbol{\beta})$ & Gamma $(\boldsymbol{\gamma})$ & $\begin{array}{l}\text { Initially Impacted } \\
\text { (\% of nodes) }\end{array}$ \\
\hline 0 & 0.025 & 0.02 & 1 \\
140 & 0.02 & 0.013 & 0.8 \\
300 & 0.03 & 0.024 & 0.5 \\
600 & 0.02 & 0.015 & 0.4 \\
\hline
\end{tabular}

diffusion under various hypothetical fluvial flooding events. A better understanding of the impact of parameters of the SIS diffusion model on the diffusion profile of the road networks is crucial as different combinations of $\beta$ and $\gamma$ values represent a different flooding profile, like the intensity of precipitation, runoff, the capacity of the flood control infrastructure or drainage systems. Furthermore, once an estimate of the values for the SIS diffusion parameters, Beta $(\beta)$ and Gamma $(\gamma)$, are obtained, it is possible to conduct scenario analysis by initiating the diffusion from different locations in the road network, which represents areas fluvial flooding most likely starts. Figure 6 (highlighted in red) shows a set of randomly chosen nodes that serve as the seed nodes for the diffusion. In order to facilitate a comparison between different scenarios, $5 \%$ of the total node number were selected for all the scenarios and diffusions are simulated in the same network in Memorial Super Neighborhood.

Figure 7 depicts the connectivity profile of the road network in the case study area under different diffusion scenarios, where diffusions are initiated from nodes with high centrality measures. The extent of diffusion was simulated until about $20 \%$ of the nodes were removed from the road network. In order to facilitate a comparison between the

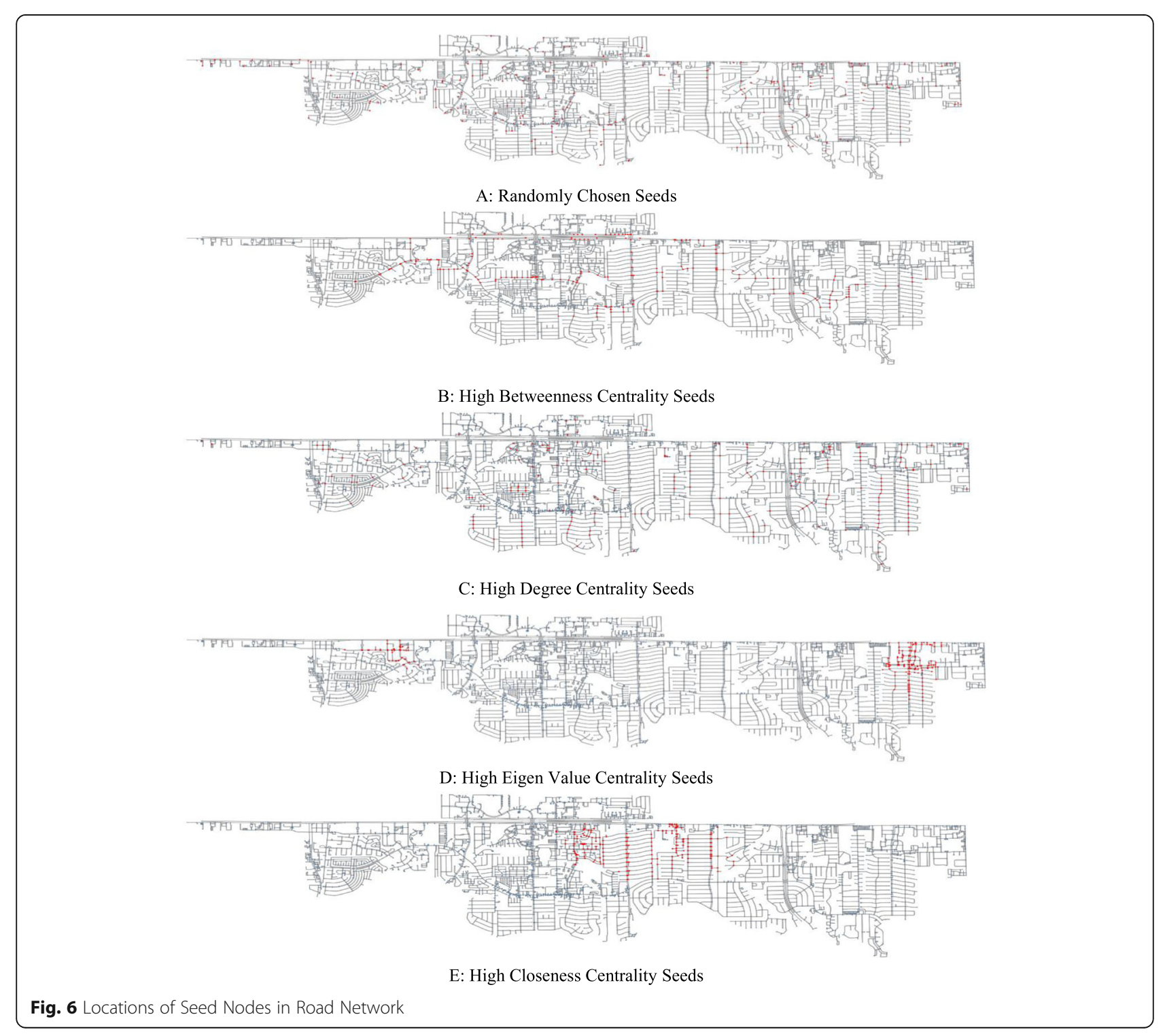




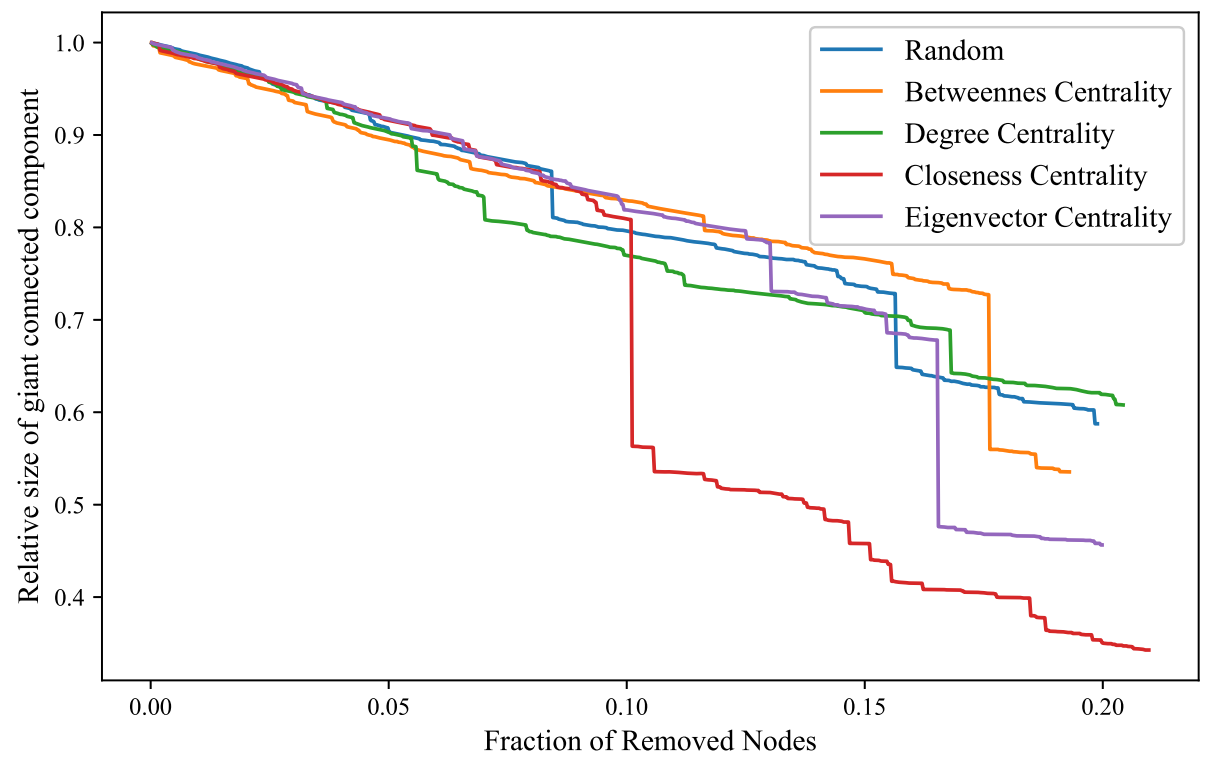

Fig. 7 Connectivity Profile of Road Network under Diffusion with Different Seed Locations

impacts of diffusive disruption and targeted disruptions on road network connectivity, the authors simulated five intentional disruptions. Figure 8 shows the connectivity profile of the road network when the nodes in the network are intentionally removed at a decreasing order of corresponding node centrality measure.

As could be seen from Fig. 7, the rate at which the connectivity of the road network is reduced under different diffusion scenarios varies significantly. There is an apparent non-linear pattern of reduction in the connectivity of road networks when diffusion in the road network is initiated from nodes with high betweenness, degree and closeness centralities. The connectivity of the road network is particularly vulnerable to the diffusion initiated from nodes with high betweenness centrality (BC), as removal of less than $5 \%$ of nodes from the network reduced the size of GCC into less than $70 \%$ of the original size. When the fraction of removed nodes reaches $10 \%$, the size of GCC becomes less than half of its original size. However, under this scenario, the impact of disruption on connectivity becomes less severe as the fraction of removed nodes increases. This is probably due to the fact that, once the significant nodes (with high $\mathrm{BC}$ values) are removed and disruption propagates

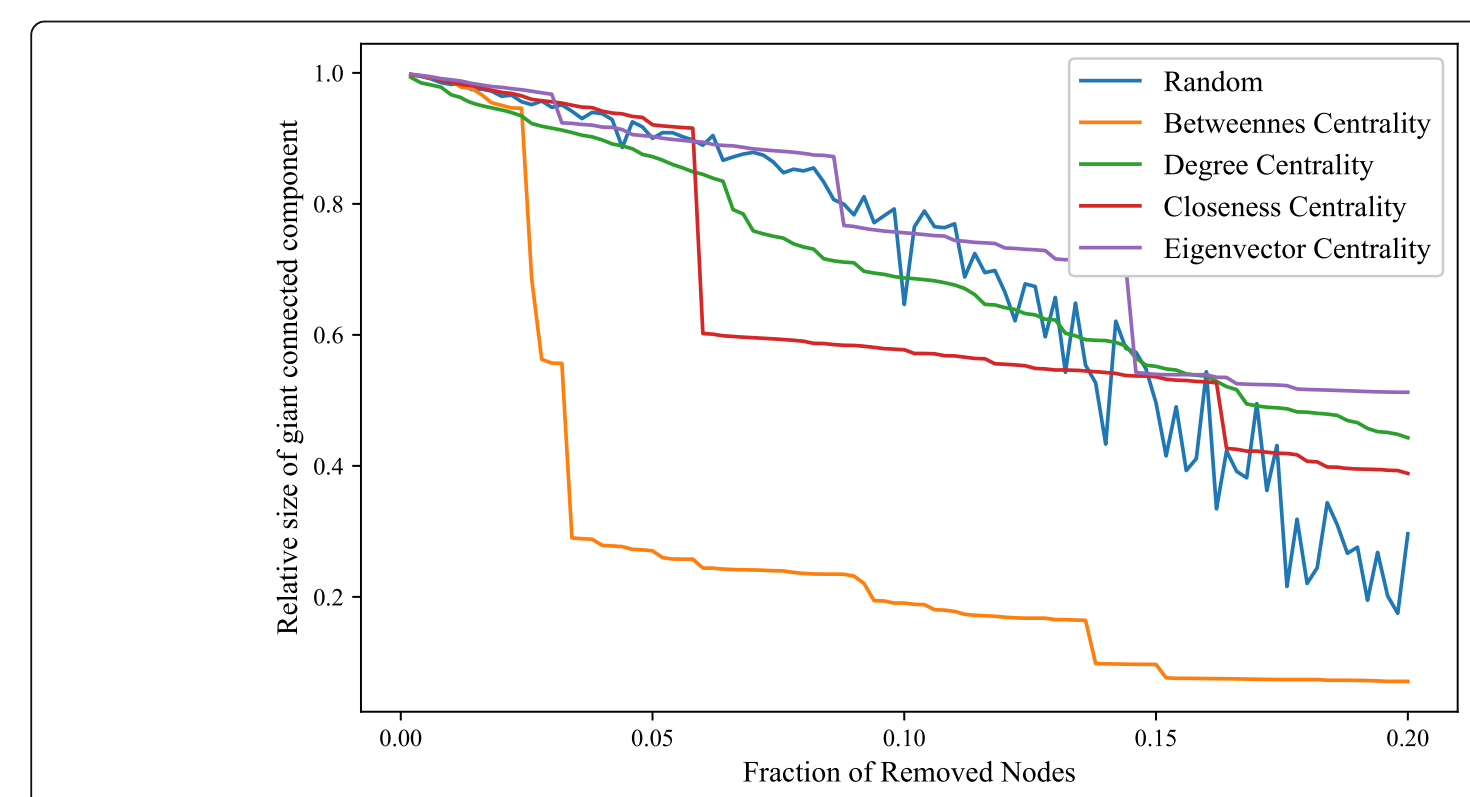

Fig. 8 Connectivity Profile of Road Network under Different Targeted Disruption Scenarios 
Table 2 Results of the hypothesis tests (on the different networks)

\begin{tabular}{llll}
\hline Initial Seed Type & Working Hypothesis & z-statistics & Conclusion (at $\boldsymbol{a}=0.1)$ \\
\hline high DC & $\mu_{A D}<\mu_{\text {random }}$ & 0.214921 & Fail to reject the Null \\
high BC & $\mu_{B C}<\mu_{\text {random }}$ & 2.078136 & Reject the Null \\
high CC & $\mu_{C C}<\mu_{\text {random }}$ & 0.677877 & Fail to reject the Null \\
high EC & $\mu_{E C}<\mu_{\text {random }}$ & 1.780124 & Reject the Null \\
\hline
\end{tabular}

into less significant nodes, further removal of nodes doesn't cause a sharp reduction on the network connectivity, which is validated by the quick reduction of connectivity under high BC scenario in Fig. 8. At different magnitudes of disruption (as the fraction of removed nodes varies in $\mathrm{x}$-axis), the overall reduction of the connectivity of the road network is also different. As the area under the high-BC curve is significantly smaller than areas under any other scenario. The particularly severe impact of nodes with high betweenness centrality on the connectivity of the road network could be observed from both Figs. 7 and 8.

\section{Experiment two: characterization of road network vulnerability to diffusive disruptions}

A separate simulation of diffusion on the road network was conducted for each of the scenarios (random, BE, $\mathrm{DC}, \mathrm{CC}$, and EC). The working hypothesis is that a diffusion starts from nodes with high centrality values will cause a greater loss in the connectivity. According to this hypothesis, the average connectivity of the road network under these scenarios $\left(\mu_{A D}, \mu_{B C}, \mu_{C C}, \mu_{E C}\right)$ is less than connectivity of the road network under a diffusive failure which starts from a set of nodes randomly chosen. The parameters of the diffusion are initially impacted seed size $\alpha(\alpha=1 \%, 5 \%$ and $10 \%), \beta=0.04, \gamma=$ 0.02 . This process was conducted for 88 super neighborhoods in Houston, in order to get the sample of the road network connectivity under these scenarios. Independence between samples was assumed, as the number of samples is more than 30 , the z-test was used for testing the hypothesis. Table 2 presents a summary of the hypothesis testing when the seed size parameter is $\alpha=10 \%$. When larger seed size values (1\% and 5\%) are used, the results for tall high centrality scenarios (high DC, EC, CC and $\mathrm{BC})$ centrality scenarios are not significantly different from the diffusion initiated from randomly chosen seeds.

As could be inferred from the results in Table 2, contrary to the initial belief, diffusion started from high significance nodes do not cause the expected greater decrease in the network connectivity. Diffusion which originates from seeds of nodes that have high betweenness and high eigenvector centrality causes greater loss to the connectivity loss when the seed size is large, compared to ones that started from randomly selected nodes. In contrast, diffusion which originates from seeds of nodes that have high closeness centrality seems to cause less loss to the connectivity of the network than a diffusion started from the randomly chosen nodes.

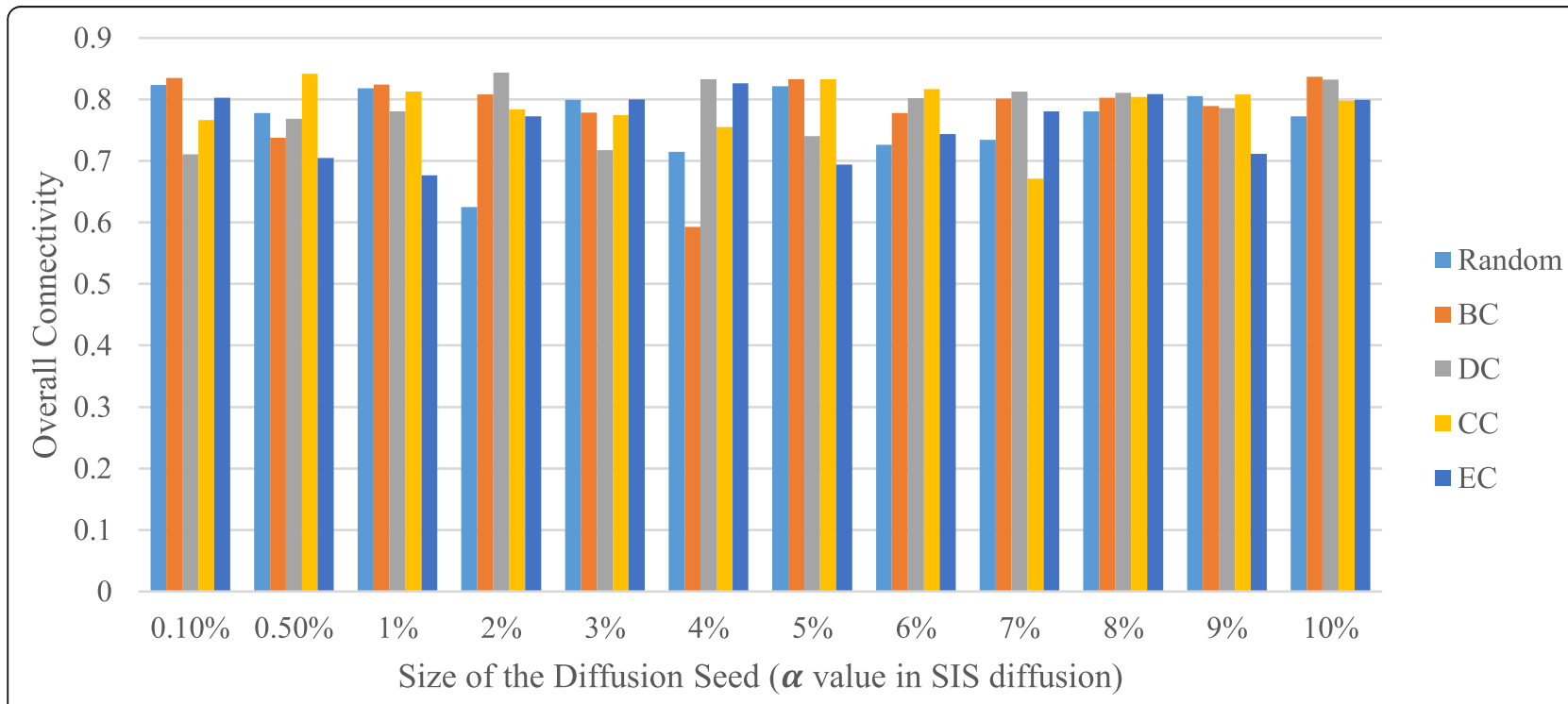

Fig. 9 Average Network Connectivity under different Diffusion Scenarios 
Average connectivities in road networks (for the above-mentioned five cases) under different seed-size scenarios were also studied (see Fig. 9). Diffusion which originates from seeds of nodes that have high eigenvector and betweenness centrality seems to cause more significant loss to the connectivity loss when the seed sizes are respectively $2 \%$ and $4 \%$. It is also observed that if diffusion starts from a larger number of nodes (above $7 \%$ of total nodes) with high betweenness centrality, the impacts on the network connectivity would be higher than that of diffusion originated from the randomly chosen nodes with the same size.

The above findings have important implications for flood management. The figures indicate that to ensure the connectivity of the graph or the transportation network, it is crucial to maintain the functionality of a number of critical nodes in the network. This paper illustrates that the SIS diffusion model can be used to identify critical nodes in transportation road networks. This paper also presented a sensitivity analysis of the impact of the number of initially flooded nodes. The second application of the finding is that network size increase may not necessarily result in improved robustness in the network. This also means that just adding extra lanes to the roadways may not improve the flood resilience of the road network. Instead, working on ensuring the functionality of a few nodes in road networks is critical to the robustness of the road network.

\section{Conclusions}

This paper presented the use of the SIS diffusion model to study diffusion phenomena in the road network under the influence of the fluvial flooding during heavy rainfall. The results show that there is significant variability in the sensitivity of the road network connectivity to the diffusive disruptions initiated from different locations. The results indicate that the road network is particularly vulnerable for disruptions that occur at nodes with high betweenness centrality. Both the diffusion-based disruptions and intentional disruptions show the variance on the impacts disruptions at different locations. It was found that a road network does have critical threshold values for the fraction of removed nodes, being above which could lead to the loss of most of the connectivity in the road network. Ensuring the fraction of removed nodes under a certain threshold could lead to disproportionate benefits in terms of the social and economic well-being of communities whose mobility depends on the network. It has been observed that the sensitivity of the robustness of the road network is different for the intentional disruptions and diffusive disruptions. It has been observed that, in general, the rate of the reduction in the connectivity is faster under the intentionally targeted disruptions than the SIS diffusive phenomenon. In summary, if we are able to predict the configuration of the road network under a given flooding scenario, then we would be able to predict various types of accessibilities. There is also a threshold value for the node removal portion for the robustness decrease, while the change in the robustness (measured in terms of the size of the largest connected component) under the random percolation is relatively moderate. The critical threshold value for the removal fraction of the nodes under the diffusion phenomenon is about $25 \%$. This has two critical implications on road network resilience. The first, marginal utility of the investment in improving the vulnerability of the road network is different at different disruption levels. The dividend, in terms of ensuring the connectivity of the road network, on investing to avoid the node-removal is particularly high for the nodes with high betweenness centrality.

Even though this study was aimed at bridging the gaps between findings in the realms of theoretical networks and performance of real-life networks. There are several areas that need further study in order to render the findings and conclusions in this study even more realistic. For example, the depth of the standing floodwater in the road network is an important indicator for it's being safe to travel or not, there could be numerous other factors that also should be taken into account for predicting safe travel speed. These factors could include vehicle conditions (tire pressure, roadworthiness etc.), condition of the pavement, visibility and aptitude and behavior of the driver during the flooding events because all of these could contribute to whether a road network is being "closed" or not. In addition, this study demonstrated the applicability of SIS diffusion model for characterizing the vulnerability of road network against fluvial flooding and results are highly specific to the case study, as the parameters of the SIS diffusion are dependant on numerous factors, like topology of the road network, spatial and temporal variability in the magnitude of flood and the temporal unit selected for the diffusion analysis. The static diffusion parameters this study estimated is an abstraction of the dynamic process in the road network. Therefore, extrapolating the results from one location (under a given flooding scenario at a given threshold) to other flooding scenario or other locations might not be feasible. In terms of the granularity of the data, this study has used the hourly flood depth data as input for the diffusion. Based on the rainfall intensity and other factors, less or more granular data could be needed in order to predict the transitions between node statuses. This process is not captured in the $S \rightarrow I \rightarrow S$ transition process. It is also possible to train diffusion models using the data for multiple super neighborhoods or under multiple types of flooding scenarios, which could enable the identification of the diffusion model that is best able to model the floodwater diffusion in road networks during a given flooding event. 


\section{Abbreviations}

SIS: Susceptible-Impacted-Susceptible; GCC: Giant Connected Component; ICl: Interdependent Critical Infrastructure; FEMA: Federal Emergency Management Administration; DEM: Digital Elevation Model; LiDAR: Light Detection and Ranging; HWM: High-Water Marks; USGS: United States Geological Survey; API: Application Programming Interfaces; TSARP: Tropical Storm Allison Recovery Project; BC: Betweenness Centrality; DC: Degree Centrality; CC: Closeness Centrality; OC: Overall Connectivity

\section{Acknowledgments}

Not applicable.

\section{Authors' contributions}

$\mathrm{BA}$ and $\mathrm{BB}$ conceived the idea together. AK and RF used hydrological models to estimate the temporal flood depth at the nodes in the road network; AK provided feedbacks to the structural organization of the manuscript; BA analyzed and interpreted the relevant data. BA performed the analysis and was a major contributor in writing the manuscript. All authors read and approved the final manuscript.

\section{Funding}

The authors would like to acknowledge funding support from the National Science Foundation RAPID project \# (1760258): RAPID: "Assessment of Risks and Vulnerability in Coupled Human-Physical Networks of Houston's Flood Protection, Emergency Response, and Transportation Infrastructure in Harvey." CRISP project \# (1832662): "Anatomy of Coupled Human-Infrastructure Systems Resilience to Urban Flooding: Integrated Assessment of Social, Institutional, and Physical Networks." Any opinions, findings, and conclusions or recommendations expressed in this research are those of the authors and do not necessarily reflect the view of the funding agency.

\section{Availability of data and materials}

Some or all data, models, or code generated or used during the study are available from the corresponding author by request.

\section{Competing interests}

The authors declare that they have no competing interests.

\section{Author details}

'Zachry Department of Civil Engineering, Texas A\&M University, 3136 TAMU, College Station, TX 77843, USA. ${ }^{2}$ Research Associate, Oden Institute for Computational Engineering and Sciences, University of Texas at Austin, Austin, TX 78712, USA. 'Environmental Engineering Graduate Program, N138 Engineering Building 1, Houston, TX 77204-4003, USA.

\section{Received: 4 December 2019 Accepted: 28 January 2020} Published online: 23 March 2020

\section{References}

1. Abdulla B, Mostafavi A, Birgisson B (2019) Characterization of the vulnerability of road networks to fluvial flooding using network percolation approach. In: Computing in Civil Engineering 2019: Smart Cities, Sustainability, and Resilience (pp. 428-435). American Society of Civil Engineers, Reston

2. ASCE. (2017). Report Card for America's Infrastructure. Retrieved May 9, 2019, from https://www.infrastructurereportcard.org/. Accessed 9 May 2019

3. Ashley ST, Ashley WS (2008) Flood fatalities in the United States. J Appl Meteorol Climatol 47(3):805-818

4. Bashan A, Berezin Y, Buldyrev SV, Havlin S (2013) The extreme vulnerability of interdependent spatially embedded networks. Nat Phys 9(10):667

5. Blackburn J (2017) Living with Houston flooding

6. Boeing G (2017) OSMnx: new methods for acquiring, constructing, analyzing, and visualizing complex street networks. Comput Environ Urban Syst 65:126-139

7. Briaud J-L, Ting FCK, Chen HC, Gudavalli R, Perugu S, Wei G (1999) SRICOS: prediction of scour rate in cohesive soils at bridge piers. J Geotech Geoenviron 125(4):237-246

8. Courty LG, Rico-Ramirez MA, Pedrozo-Acuna A (2017) The significance of infiltration and spatial variability of rainfall on the numerical reproduction of urban floods
9. Drobot SD, Benight C, Gruntfest EC (2007) Risk factors for driving into flooded roads. Environ Hazards 7(3):227-234

10. Dueñas-Osorio L, Craig Jl, Goodno BJ, Bostrom A (2007) Interdependent response of networked systems. J Infrastruct Syst 13(3):185-194

11. FitzGerald G, Du W, Jamal A, Clark M, Hou X (2010) Flood fatalities in contemporary Australia (1997-2008). Emerg Med Australas 22(2):180-186

12. Haimes $Y Y$, Jiang $P$ (2001) Leontief-based model of risk in complex interconnected infrastructures. J Infrastruct Syst 7(1):1-12

13. National Academy of Engineering (2002) Frontiers of Engineering: Reports on Leading-Edge Engineering from the 2001 NAE Symposium on Frontiers of Engineering. The National Academies Press, Washington, DC. https://doi. org/10.17226/10494

14. Kalantari Z, Cavalli M, Cantone C, Crema S, Destouni G (2017) Flood probability quantification for road infrastructure: data-driven spatialstatistical approach and case study applications. Sci Total Environ 581:386398

15. Kiaghadi A, Rifai HS (2019) Physical, chemical, and microbial quality of floodwaters in Houston following hurricane Harvey. Environ Sci Technol 53(9):4832-4840

16. Kreibich H, Piroth $K$, Seifert I, Maiwald H, Kunert U, Schwarz J, ... Thieken AH (2009). Is flow velocity a significant parameter in flood damage modelling?

17. Lagmay AM, Mendoza J, Cipriano F, Delmendo PA, Lacsamana MN, Moises MA et al (2017) Street floods in metro Manila and possible solutions. J Environ Sci 59:39-47

18. Leu G, Abbass H, Curtis N (2010) Resilience of ground transportation networks: a case study on Melbourne

19. Melville BW, Coleman SE (2000) Bridge scour. Water Resources Publication

20. Mark Newman. 2010. Networks: An Introduction. Oxford University Press, Inc., USA

21. Mark Newman. 2018. Networks. Oxford university press., Inc., USA

22. O'Rourke TD (2007) Critical infrastructure, interdependencies, and resilience. Bridge Wash Natl Acad Eng 37(1):22

23. Pregnolato M, Ford A, Wilkinson SM, Dawson RJ (2017) The impact of flooding on road transport: a depth-disruption function. Transp Res Part D: Transp Environ 55:67-81

24. Pyatkova K, Chen AS, Butler D, Vojinović Z, Djordjević S (2019) Assessing the knock-on effects of flooding on road transportation. J Environ Manag 244: 48-60

25. Reed DA, Kapur KC, Christie RD (2009) Methodology for assessing the resilience of networked infrastructure. IEEE Syst J 3(2):174-180

26. Rodin J (2014) The resilience dividend: being strong in a world where things go wrong. Gildan Media, LLC

27. Rossetti G, Milli L, Rinzivillo S, Sîrbu A, Pedreschi D, Giannotti F (2018) NDlib: a python library to model and analyze diffusion processes over complex networks. Int J Data Sci Analytics 5(1):61-79

28. Sadler JM, Haselden N, Mellon K, Hackel A, Son V, Mayfield J et al (2017) Impact of sea-level rise on roadway flooding in the Hampton roads region, Virginia. J Infrastruct Syst 23(4):5017006

29. Schneider CM, Moreira AA, Andrade JS, Havlin S, Herrmann HJ (2011) Mitigation of malicious attacks on networks. Proc Natl Acad Sci 108(10): 3838-3841

30. Shakarian P, Bhatnagar A, Aleali A, Shaabani E, Guo R (2015) Diffusion in social networks. Springer. Retrieved from. https://doi.org/10.1007/978-3-31923105-1.pdf

31. Singh P, Sinha VSP, Vijhani A, Pahuja N (2018) Vulnerability assessment of urban road network from urban flood. Int J Disaster Risk Reduction 28:237250

32. Tamvakis $P$, Xenidis $Y$ (2013) Comparative evaluation of resilience quantification methods for infrastructure systems. Procedia Soc Behav Sci 74:339-348

33. van Laere J, Berggren P, Gustavsson P, Ibrahim O, Johansson B, Larsson A et al (2017) Challenges for critical infrastructure resilience: cascading effects of payment system disruptions, In 14th International Conference on Information Systems for Crisis Response and Management (ISCRAM2017), vol 14. ISCRAM, Albi, France, pp 281-292

34. Wang C, Yu X, Liang F (2017) A review of bridge scour: mechanism, estimation, monitoring and countermeasures. Nat Hazards 87(3):1881-1906

\section{Publisher's Note}

Springer Nature remains neutral with regard to jurisdictional claims in published maps and institutional affiliations. 